\title{
Guyer-Krumhansl heat conduction in thermoreflectance experiments
}

\author{
Matthew G. Hennessy and Timothy G. Myers
}

\begin{abstract}
Thermoreflectance experiments involve heating the surface of a solid using a high-frequency laser. The small length and time scales associated with this rapid heating lead to the onset of heat conduction mechanisms that cannot be captured using Fourier's law. We propose a model for thermoreflectance experiments based on the Guyer-Krumhansl equation of heat conduction. We show that heat conduction occurs in the form of two distinct modes which are analogous to pressure and shear waves in linear viscoelastic materials. We present analytical solutions to the model that can be used to calculate the three-dimensional temperature and flux profiles in the heated solid as well as the phase difference between the laser and the surface temperature oscillations. Using the Laplace transform, we show how the solution can be extended to account for laser pulses with an arbitrary dependence on time.
\end{abstract}

\section{Introduction}

Nanotechnology is a rapidly growing interdisciplinary area, with breakthroughs having important implications in fields such as medicine, electronics, and energy storage. One of the key issues with designing nanodevices is inefficient dissipation of heat, which can lead to performance degradation, melting, and ultimately device failure $[1,2]$. Theoretical and experimental studies have shown that there is a marked decrease in the thermal conductivity at the nanoscale $[3,4,5]$, which prevents generated heat from being dissipated or exchanged with the surrounding environment. As the characteristic size of the system decreases, heat conduction becomes increas-

Matthew G. Hennessy

Mathematical Institute, University of Oxford, Andrew Wiles Building, Woodstock Road, Oxford, OX2 6GG, United Kingdom e-mail: hennessy@ maths.ox.ac.uk

Timothy G. Myers

Centre de Recerca Matemàtica, Campus de Bellaterra, Edifici C, 08193 Bellaterra, Spain e-mail: tmyers@crm.cat 
ingly dominated by the infrequency of collisions between thermal energy carriers known as phonons. The finite time between successive phonon collisions results in a ballistic transport process which effectively reduces the thermal conductivity of the system. Conversely, classical heat conduction is a diffusive process that occurs when the characteristic length and time scales are much larger than the mean free path and mean free time of phonon collisions.

A number of experiments have been designed to access the extremely small length and time scales associated with nanoscale heat transport. In thermoreflectance experiments, a high-frequency laser (called the pump beam) is used to heat the surface of a metallic film that is bonded to a sample of semiconducting material such as silicon or germanium [6,7]. A secondary laser (called the probe beam) is used to measure the change in reflectance of the metal film. From these measurements, the surface temperature of the bilayer can be deduced and compared to theoretical predictions. Unsurprisingly, models based on Fourier's law which use the bulk value of the thermal conductivity are in poor agreement with experimental data as a consequence of the ballistic nature of nanoscale heat conduction not being accounted for. It is therefore common to interpret thermoreflectance experiments through the use of effective Fourier models (EFMs), which introduce features such as anisotropic thermal conductivity tensors [8] or frequency-dependent material properties [7] in order to match the experimental data. Although EFMs provide important insights into thermoreflectance experiments, they are bound to the assumption of diffusive transport and, as a result, can fail to provide consistent interpretations of the data. For example, fitting an EFM to data of the amplitude of oscillations in the surface temperature results in a thermal conductivity that monotonically increases with frequency; however, fitting to data of the phase of the oscillations leads to a conductivity that monotonically decreases with frequency [9].

The failure of Fourier's law to capture thermoreflectance data motivates the need for extended models that better capture the ballistic nature of nanoscale heat conduction. The first extension to Fourier's law was proposed by Cattaneo [10], who argued that a finite amount of time is required for a temperature gradient to produce a flux of thermal energy. By expanding the governing equations about a small delay time, the Maxwell-Cattaneo (MC) equation (or Maxwell-CattaneoVernotte) equation can be obtained. When the MC equation is combined with the law of conservation of energy, the hyperbolic heat equation (HHE) is obtained, which captures the wave-like propagation of heat associated with ballistic transport. Although the HHE produces wave-like solutions, the ad-hoc introduction of a delay time does little to explain the underlying physics of heat conduction. Guyer and Krumhansl $[11,12]$ later derived an extension to the MC equation directly from the linearised Boltzmann equation that explicitly accounts for the mean free time and mean free path of phonon collisions. From a theoretical point of view, the Guyer-Krumhansl (GK) equation is particularly attractive because it provides a link between kinetic and continuum models and is based parameters that have a welldefined meaning in terms of the underlying physics of heat conduction. Moreover, the analogous form of the GK and Navier-Stokes equations enables nanoscale heat 
transport to be interpreted in terms of fluid mechanics and this analogy has been used to explain the reduced thermal conductivity of nanosystems [13, 14].

The thermal response of a solid that is heated by a laser has been studied using non-Fourier conduction laws by several authors. Zhang et al. [15] considered a threedimensional axisymmetric problem where the thermal flux was assumed to satisfy the MC equation. Maassen and Lundstrom [16] considered a one-dimensional situation and compared results obtained from the MC equation to those from the lattice Boltzmann equation (LBE), showing the two approaches are in excellent agreement. Kovács [17] also considered a one-dimensional geometry and modelled heat flow using the GK equation. Beardo et al. [9] studied three-dimensional heat conduction in thermoreflectance experiments using a model based on the GK equation which accounted for the thin metal film that is bonded to the surface of a semiconductor. They developed an analytical solution by assuming that the laser heating is harmonic in time.

In this paper we also model thermoreflectance experiments using the GK equation. We neglect the thin metal film used in experiments but allow for the laser heating to have an arbitrary dependence on time. We show that the full unsteady form of the thermal model is analogous to the Kelvin-Voigt model of a linear viscoelastic material. We use this analogy to decompose the solution of the thermal model into modes that mimic viscoelastic pressure and shear waves.

The outline of the paper is as follows. In Sec. 2 we present the governing equations. In Sec. 3 the equations are related to those of linear viscoelasticity and the solution is decomposed into wave-like modes. In Sec. 4, semi-analytical and analytical solutions are constructed when the laser heating is harmonic in time. In Sec. 5 we discuss how the previous solutions can be extended to account for arbitrary forms of the laser heating through the use of Laplace transforms. The paper then concludes in Sec. 6.

\section{Mathematical model}

We consider the heating of an isotropic solid sample with a high-frequency laser as shown in Figure 1. Conservation of energy within the sample requires

$$
c_{v} \dot{T}+\nabla \cdot \boldsymbol{q}=0
$$

where $c_{v}$ is the volumetric heat capacity (at constant volume) of the sample, $T$ is the temperature difference relative to the initial and ambient temperature $T_{0}$, and $\boldsymbol{q}=\left(q_{x}, q_{y}, q_{z}\right)$ is the thermal flux. It is assumed that the optical extinction length is sufficiently small that the absorption of radiation can be captured through a boundary condition rather than a volumetric heating term in (1). The thermal flux is assumed to obey the Guyer-Krumhansl (GK) equation

$$
\tau_{R} \dot{\boldsymbol{q}}+\boldsymbol{q}=-k_{\mathrm{bulk}} \nabla T+\ell^{2}\left[\nabla^{2} \boldsymbol{q}+2 \nabla(\nabla \cdot \boldsymbol{q})\right],
$$


where $\tau_{R}, k_{\text {bulk }}$, and $\ell$ are the relaxation time of resistive phonon collisions, bulk thermal conductivity, and bulk mean free path (MFP) of phonons in the sample. Mathematically, the first term on the left-hand side of (2) accounts for memory effects and leads to the thermal flux depending on the time integral of the temperature gradient. The second and third terms on the right-hand side, i.e. those proportional to $\ell^{2}$, represent non-local effects and lead to the flux depending on the spatial integral of the temperature gradient. The thermal conductivity and MFP can be written as $k_{\text {bulk }}=(1 / 3) c_{v} v_{g}^{2} \tau_{R}$ and $\ell^{2}=(1 / 5) v_{g}^{2} \tau_{R} \tau_{N}$ with $v_{g}$ being the speed of phonons in the material and $\tau_{N}$ the relaxation time of normal phonon collisions. Furthermore, we introduce the bulk thermal diffusivity $\kappa=k_{\text {bulk }} / c_{v}$.

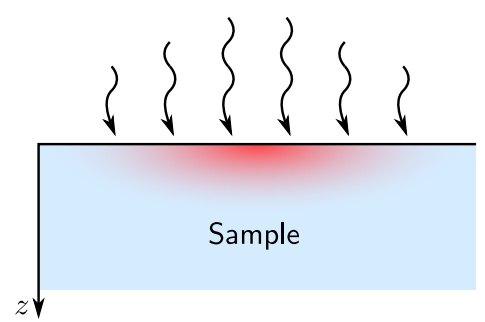

Fig. 1 A schematic diagram of a thermoreflectance experiment whereby the surface of a solid is heated by a high-frequency laser with a Gaussian intensity distribution.

The laser induces a thermal flux at the irradiated surface of the solid given by

$$
\boldsymbol{q} \cdot \boldsymbol{e}_{z}=q_{0} I(x, y) f(t), \quad z=0,
$$

where $\boldsymbol{e}_{z}$ is the unit vector normal to the surface with the coordinate $z$ pointing into the sample; $I$ describes the non-dimensional in-plane distribution of the laser intensity; $x$ and $y$ are horizontal coordinates centred at the point of irradiation; $f$ describes the temporal aspects of the laser pulse; and $t$ is time. We will assume that the beam profile is Gaussian and given by

$$
I(x, y)=\mathrm{e}^{-2\left(x^{2}+y^{2}\right) / r_{b}^{2}},
$$

where $r_{b}$ is the $1 / \mathrm{e}^{2}$ radius of the beam and $q_{0}$ represents the maximum intensity of the laser, which is related to the laser power $P$ through the relation $q_{0}=P /\left(\pi r_{b}^{2}\right)$.

Following previous works $[18,19,20]$, slip-like boundary conditions for the tangential components of the thermal flux are specified,

$$
q_{x}=C l \frac{\partial q_{x}}{\partial z}, \quad q_{y}=C l \frac{\partial q_{y}}{\partial z} ; \quad z=0 .
$$

The dimensionless parameter $C$ encodes information about the nature of phonon scattering at the boundary, surface roughness, and temperature effects [13, 20, 21]. 
Here we make the assumption that phonons undergo diffusive scattering at the boundary and set $C=1$.

We also impose far-field conditions of the form $T / T_{0} \rightarrow 0$ and $q / q_{0} \rightarrow 0$ as $z / L_{F} \rightarrow \infty$, where $L_{F}=(2 \kappa / \omega)^{1 / 2}$ is the classical thermal penetration depth predicted with Fourier's law. We also assume that $T=0$ and $\boldsymbol{q}=0$ when $t=0$.

The parameter values are based on the thermoreflectance experiments in Regner et al. [6, 7] involving c-Si. In these experiments, the radius of the pump beam was $r_{b}=3.2 \mu \mathrm{m}$. When required, we assume that the power of the pump beam is $P=1 \mathrm{~mW}$. The thickness of the layer (in the $z$ direction) was $H=525 \mu \mathrm{m}$. Since the thickness of the sample is much greater than the maximum thermal penetration depth, $H / L_{F} \gg 1$, we assume that the sample can be treated as infinitely thick. The temperature-dependent parameter values are listed Table 1 . The resistive relaxation time and MFP are taken from Beardo et al. [9].

Table 1 Temperature-dependent parameter values.

\begin{tabular}{l|cc}
\hline Parameter [unit] & $81 \mathrm{~K}$ & $311 \mathrm{~K}$ \\
\hline$c_{v}\left[\mathrm{KJ} \mathrm{m}^{-3} \mathrm{~K}^{-1}\right]$ & 466 & 1692 \\
$k_{\text {bulk }}\left[\mathrm{W} \mathrm{m}^{1} \mathrm{~K}^{-1}\right]$ & 1260 & 150 \\
$\tau[\mathrm{ps}]$ & 1002 & 42 \\
$\ell[\mathrm{nm}]$ & 3127 & 185
\end{tabular}

\section{Connections to linear viscoelasticity}

Elimination of the temperature $T$ from (2) shows that the thermal flux satisfies

$$
\tau_{R} \ddot{\boldsymbol{q}}+\dot{\boldsymbol{q}}=\kappa \nabla(\nabla \cdot \boldsymbol{q})+\ell^{2}\left[\nabla^{2} \dot{\boldsymbol{q}}+2 \nabla(\nabla \cdot \dot{\boldsymbol{q}})\right]
$$

The bulk equation (6) and the boundary condition (3) show that the governing equations for the thermal flux are the same as those of a damped Kelvin-Voigt viscoelastic material with zero shear modulus that is subject to a fixed surface displacement. More specifically, the resistive relaxation time is analogous to the density; the thermal diffusivity is the Lamé parameter; and the square of the MFP is the viscosity (shear and bulk).

Motivated by the analogy between nanoscale heat transfer and linear viscoelastic materials, we introduce a Helmholtz decomposition of the flux,

$$
\boldsymbol{q} / q_{0}=\nabla \Phi+\nabla \times \boldsymbol{A}
$$

in order to simplify the bulk equations given in (6). The scalar and vector potentials $\Phi$ and $\boldsymbol{A}$, respectively, can be shown to satisfy 


$$
\begin{aligned}
\tau_{R} \ddot{\Phi}+\dot{\Phi} & =\kappa \nabla^{2} \Phi+3 \ell^{2} \nabla^{2} \dot{\Phi}, \\
\tau_{R} \dot{\boldsymbol{A}}+\boldsymbol{A} & =\ell^{2} \nabla^{2} \boldsymbol{A}, \\
\nabla \cdot \boldsymbol{A} & =0 .
\end{aligned}
$$

In the context of linear elasticity, the scalar and vector potentials capture pressure $(\mathrm{P})$ and shear (S) waves, respectively. Both waves are non-dispersive; however, P-waves are longitudinal and irrotational, whereas $\mathrm{S}$-waves are transverse and incompressible (i.e. volume conserving). In the context of heat flow, $\mathrm{S}$-waves represent temperatureconserving thermal waves which propagate in orthogonal directions to the flux and do not influence the temperature. Only P-waves can generate a change in temperature, which, mathematically, can be seen by combining (1) and (7) to obtain $c_{v} \dot{T}=$ $-q_{0} \nabla^{2} \Phi$. In the classical limit, $\omega \tau_{R} \rightarrow 0$ and $\ell / L_{F} \rightarrow 0$, we find that $\boldsymbol{A}=0$ and thus the scalar potential simply becomes a multiple of the temperature, $\Phi=-\left(k_{\text {bulk }} / q_{0}\right) T$.

The problem for the vector potential can be simplified by introducing the "streamfunction" $\psi$ and writing $\boldsymbol{A}=\nabla \times\left(\psi \boldsymbol{e}_{z}\right)$. This form of $\boldsymbol{A}$ automatically fulfils the condition (8c). Moreover, the problem for $\psi$ is given by

$$
\nabla^{2}\left(\tau_{R} \dot{\psi}+\psi-\ell^{2} \nabla^{2} \psi\right)=0
$$

To make further progress on the problem, we must specify the form of the heating function $f(t)$ in (3).

\section{Time-harmonic heating}

The laser pulses are first assumed to be harmonic in time, $f(t)=\mathrm{e}^{-i \omega t}$, where $\omega$ is the angular frequency of the laser. The problem can be simplified by first taking the two-dimensional (in-plane) Fourier transform of the governing equations and then seeking separable solutions of the form $\tilde{g}(\boldsymbol{k}) \mathrm{e}^{-\lambda(\boldsymbol{k}) z-i \omega t}$, where $\boldsymbol{k}=\left(k_{x}, k_{y}, 0\right)$ is a two-dimensional wavevector. The two-dimensional inverse Fourier transforms can be converted into one-dimensional Hankel transforms due to the axisymmetry of the problem, which can then be numerically evaluated using fast methods [22]. The scalar potential and streamfunction are given by

$$
\begin{aligned}
& \Phi(r, z, t)=\int_{0}^{\infty} \tilde{\Phi}(k) \tilde{I}(k) \mathrm{e}^{-\lambda_{p}(k) z-i \omega t} J_{0}(k r) k \mathrm{~d} k, \\
& \psi(r, z, t)=\int_{0}^{\infty} \tilde{\psi}(k) \tilde{I}(k) \mathrm{e}^{-\lambda_{s}(k) z-i \omega t} J_{0}(k r) k \mathrm{~d} k,
\end{aligned}
$$

where $r=\left(x^{2}+y^{2}\right)^{1 / 2}, k=|\boldsymbol{k}|$, and $\tilde{I}$ is the Hankel transform of the Gaussian beam profile in (4). The functions $\lambda_{p}$ and $\lambda_{s}$ are defined as 


$$
\begin{aligned}
& \lambda_{p}(k)=\left\{k^{2}-\frac{2 i\left(1-i \omega \tau_{R}\right)}{L_{F}^{2}\left[1-6 i\left(\ell / L_{F}\right)^{2}\right]}\right\}^{1 / 2}, \\
& \lambda_{s}(k)=\left\{k^{2}+\ell^{-2}\left(1-i \omega \tau_{R}\right)\right\}^{1 / 2},
\end{aligned}
$$

with $\operatorname{Re}\left\{\lambda_{s}\right\}>0$ and $\operatorname{Re}\left\{\lambda_{p}\right\}>0$. The penetration depths of the thermal Pand $S$-modes are given by $L_{\mathrm{P}}=1 / \operatorname{Re}\left\{\lambda_{p}(0)\right\}$ and $L_{\mathrm{S}}=1 / \operatorname{Re}\left\{\lambda_{s}(0)\right\}$. The ratio $\ell / L_{F}$ defines an effective Knudsen number, characterising the relevance of nonlocal effects when the characteristic length scale of the system is $L_{F}$. The Fourier coefficients $\tilde{\Phi}$ and $\tilde{\psi}$ are obtained from the boundary conditions (3) and (5), resulting in

$$
\begin{gathered}
\tilde{\Phi}(k)=\frac{\lambda_{s}\left(1+C \ell \lambda_{s}\right)}{k^{2}\left(1+C \ell \lambda_{p}\right)-\lambda_{s} \lambda_{p}\left(1+C \ell \lambda_{s}\right)}, \\
\tilde{\psi}(k)=\frac{1+C \ell \lambda_{p}}{k^{2}\left(1+C \ell \lambda_{p}\right)-\lambda_{s} \lambda_{p}\left(1+C \ell \lambda_{s}\right)},
\end{gathered}
$$

The temperature is given by

$$
\begin{aligned}
T(r, z, t) & =\int_{0}^{\infty} \tilde{T}(k) \tilde{I}(k) \mathrm{e}^{-\lambda_{p}(k) z-i \omega t} J_{0}(k r) k \mathrm{~d} k, \\
\tilde{T}(k) & =-\frac{q_{0}}{k_{\text {bulk }}}\left(\frac{1-i \omega \tau_{R}}{1-6 i\left(\ell / L_{F}\right)^{2}}\right) \tilde{\Phi} .
\end{aligned}
$$

which can be simplified to

$$
T(r, z, t)=-\frac{q_{0}}{k_{\mathrm{bulk}}}\left(\frac{1-i \omega \tau_{R}}{1-6 i\left(\ell / L_{F}\right)^{2}}\right) \Phi(r, z, t) .
$$

The latter form provides a direct link between the temperature and the amplitude of $\mathrm{P}$-waves in the case of GK conduction. In the case of Fourier conduction, we find that $\lambda_{p}=\left(k^{2}-2 i L_{F}^{-2}\right)^{1 / 2}, \tilde{\Phi}=-\lambda_{p}^{-1}$, and $\tilde{T}=-\left(q_{0} / k_{\text {bulk }}\right) \tilde{\Phi}$.

Figure 2 shows the two-dimensional temperature profiles at a given time $t_{*}$ obtained using Fourier's law and the GK equation when the ambient temperature is $T_{0}=81 \mathrm{~K}$. We take $f=10^{8} \mathrm{~Hz}$, which leads to $L_{F} \simeq 2.9 \mu \mathrm{m}, \omega \tau_{R} \simeq 0.63$, and $\ell / L_{F} \simeq 1.07$. The time $t_{*}$ is chosen so that the real part of the temperature solution (13) evaluated at the origin is maximised. Mathematically, $t_{*}$ is the solution to $\max _{0 \leq t \leq 2 \pi / \omega} \operatorname{Re}\{T(0,0, t)\}$. In the case of Fourier's law, the isotherms are roughly elliptical, indicating that the temperature monotonically decreases with distance from the origin. When the GK equation is used, the isotherms become more complex and indicate that the temperature is non-monotonic in the radial and axial directions. Furthermore, there is a region near the illuminated surface $(z=0)$ where the temperature becomes negative for intermediate values of $r / L_{F}$. This region is seen more clearly in Figure 3, which plots the surface temperature profiles at various times. The profiles in the case of GK conduction correspond to standing waves that 
have been spatially damped due to the inclusion of non-local effects (or 'thermal viscosity'). Interestingly, this dampening leads to a localisation of the surface heating; the temperature increase is more confined to where the laser hits the surface (note that $\left.r_{b} / L_{F} \simeq 0.77\right)$ compared to the Fourier case. As a result of this localisation, the amplitude of the surface temperature oscillations is greater in the case of GK conduction.

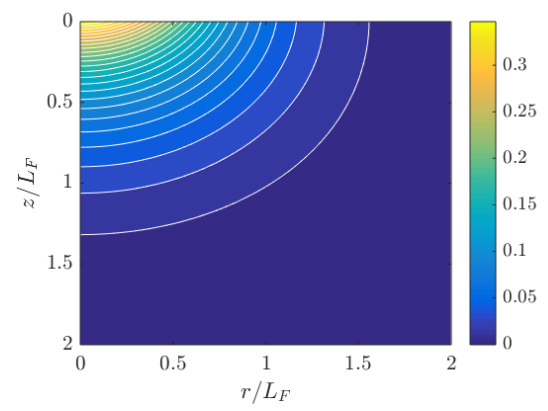

(a) Fourier conduction

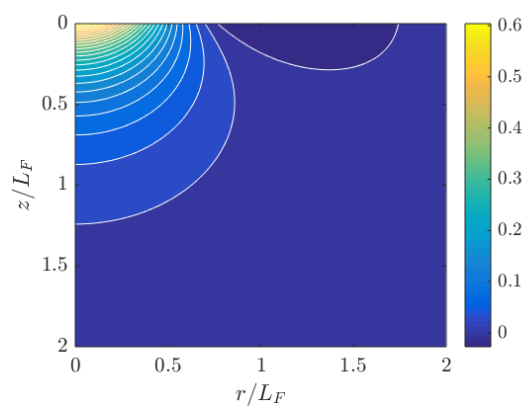

(b) GK conduction

Fig. 2 Temperature profiles obtained using Fourier's law (a) and the GK equation (b) when $T_{0}=$ $81 \mathrm{~K}$ and $f=10^{8} \mathrm{~Hz}$. The temperature has been normalised by $q_{0} L_{F} / k_{\text {bulk }}$.

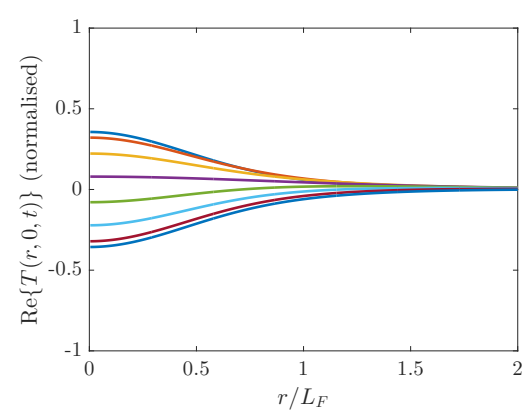

(a) Fourier conduction

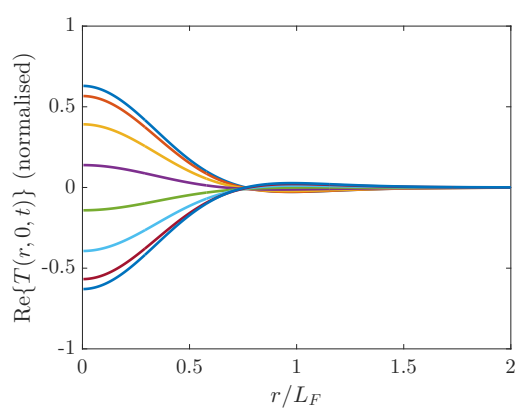

(b) GK conduction

Fig. 3 Time evolution of the surface temperature obtained using Fourier's law (a) and the GK equation (b) when $T_{0}=81 \mathrm{~K}$ and $f=10^{8} \mathrm{~Hz}$. The temperature has been normalised by $q_{0} L_{F} / k_{\text {bulk }}$. The solutions are shown at times $t=t_{*}+n \pi /(7 \omega)$ where $n=0,1, \ldots, 7$. The definition of $t_{*}$ can be found in the main body of the paper.

In thermoreflectance experiments, a probe beam is used to measure the change in reflectivity of the sample. This enables a spatially integrated form of the surface temperature to be inferred. Following Cahill [23], the surface temperature measured from a probe beam with the same profile as the pump beam can be calculated as 


$$
\Delta T_{m}=\frac{r_{b}^{2}}{4} \int_{0}^{\infty} \tilde{T}(k) \mathrm{e}^{-r_{b}^{2} k^{2} / 4} k \mathrm{~d} k .
$$

Another key experimentally measureable quantity is the phase difference between the oscillations of the surface temperature and the radiation provided by the pump beam. This phase difference can be be calculated from (15) as $\varphi=-\arg \left\{\Delta T_{m}\right\}$.

To study the dependence of the measured temperature $\Delta T_{m}$ and the phase difference $\varphi$ on frequency, we first assume that the MFP of phonons, $\ell$, is smaller than the beam radius, $r_{b}$, which is true when the ambient temperature is $T_{0}=311 \mathrm{~K}$. For small angular frequencies that satisfy $\omega \ll \kappa / r_{b}^{2}$ with $\omega \tau_{R} \ll 1$ and $\ell / L_{F} \ll 1$, the measured temperature coincides with the low-frequency Fourier-based prediction,

$$
\Delta T_{m} \simeq \Delta T_{\mathrm{LF}}=\frac{1}{2 \pi^{1 / 2}} \frac{P}{k_{\mathrm{bulk}} r_{b}} .
$$

In this case, the surface temperature is in phase with the pumb beam, $\varphi \simeq 0$ and heat is conducted in all three dimensions.

For larger frequencies that satisfy $\omega \gg \kappa / r_{b}^{2}$, the classical penetration depth $L_{F}$ is much smaller than the beam radius $r_{b}$ and heat conduction primarily occurs in the axial direction. In this case, the measured temperature can be approximated the solution obtained from a one-dimensional model,

$$
\Delta T_{m} \simeq \Delta T_{\mathrm{HF}}\left(\frac{1-i \omega \tau_{R}}{1-6 i\left(\ell / L_{F}\right)^{2}}\right)^{1 / 2}, \quad \Delta T_{\mathrm{HF}}=\frac{P}{2 \pi r_{b}^{2}}\left(\frac{i}{k_{\text {bulk }} \rho c_{\nu} \omega}\right)^{1 / 2},
$$

The prefactor $\Delta T_{\mathrm{HF}}$ corresponds to the high-frequency limit in the case of Fourier conduction. The corresponding phase shift is given by

$$
\varphi \simeq-\frac{\pi}{4}+\frac{1}{2} \arctan \left(\omega \tau_{R}\right)-\frac{1}{2} \arctan \left(\frac{6 \ell^{2}}{L_{F}^{2}}\right) .
$$

The first component, $\varphi_{\mathrm{HF}}=-\arg \left\{\Delta T_{\mathrm{HF}}\right\}=-\pi / 4$, is a frequency-independent contribution from Fourier conduction. The second and third contributions are attributed to resistive and normal phonon scattering modes, respectively. For values of the angular frequency given by $\omega \gg \tau_{R}^{-1}$ and $\omega \gg \kappa / \ell^{2}$, the one-dimensional approximation given by (17) simplifies even further to $\Delta T_{m} / \Delta T_{\mathrm{HF}} \sim\left(\tau_{R} \kappa\right)^{1 / 2} / \ell$, which indicates that the measured temperature retains a scaling that is proportional to $\omega^{-1 / 2}$. Moreover, the non-Fourier contributions to the phase shift (18) cancel out in the high-frequency limit and the Fourier result $\varphi \simeq-\pi / 4$ is recovered. In the special case when $\tau_{R} \kappa / \ell^{2}=1$, no distinction can be made between Fourier and GK conduction, a phenomenon that is sometimes referred to as Fourier resonance [24, 25]. Differences in the magnitude of $\tau_{R} \kappa / \ell^{2}$ lead to distinct behaviour in the regime of intermediate frequencies. When $\tau_{R} \ll \ell^{2} / \kappa$, which is the case for the parameters in Table 1 , there is a range of frequencies given by $\kappa / \ell^{2} \ll \omega \ll \tau_{R}^{-1}$ where the measured temperature $\left|\Delta T_{m}\right|$ scales like $\omega^{-1}$ and thus decreases faster with frequency than predicted by Fourier's law. Furthermore, the phase difference decreases be- 
yond the Fourier value and approaches $\varphi \simeq-\pi / 2$. This is shown more clearly in Figure 4 (a)-(b), in which $\left|\Delta T_{m}\right|$ and $\varphi$ are plotted as a function of frequency. Conversely, if $\tau_{R} \gg \ell^{2} / \kappa$, then there is a range of frequencies given by $\tau_{R}^{-1} \ll \omega \ll \kappa / \ell^{2}$ where $\left|\Delta T_{m}\right|$ exhibits no dependence on the frequency, leading to a higher temperature than predicted by Fourier's law, consistent with the numerical results obtained from the HHE and phonon LBE [16]. In this case, the phase difference begins to increase and approach $\varphi=0$.

In Figure 4 (a)-(b) we also compare the amplitude of the measured temperature $\left|\Delta T_{m}\right|$ and the phase difference obtained from Fourier's law, the GK equation, and the one-dimensional GK solutions given by (17) and (18) when $T_{0}=311 \mathrm{~K}$. From these figures we can deduce the existence of three distinct regimes of heat conduction at this temperature. At low frequencies, heat conduction is three dimensional and diffusive, that is, it can be captured using Fourier's law. As the frequency is increased, nonlocal effects become relevant and three-dimensional GK conduction begins to occur. However, for very high frequencies, heat conduction becomes one dimensional. The plateau in the phase difference that occurs when $f=10^{9} \mathrm{~Hz}$ indicates that resistive phonon scattering is also beginning to become relevant.

Figures 4 (c)-(d) illustrate the frequency dependence of $\left|\Delta T_{m}\right|$ and $\varphi$ when $T_{0}=$ $81 \mathrm{~K}$. In this case, the phonon MFP $\ell$ is roughly the same size as the beam radius. As a result, there are substantial deviations between the Fourier and GK temperature amplitudes even at low frequencies. Moreover, the phase difference reveals that heat conduction remains three dimensional across the entire frequency band.

\section{Arbitrary heating functions}

Using Laplace transforms, it is possible to construct an analytical solution for the temperature for arbitrary forms of the heating function $f(t)$. We let $\bar{f}(s)=\mathcal{L}\{f(t)\}$ denote the Laplace transform of $f$. Then the solution for the temperature is given by $T(r, z, t)=\mathcal{L}^{-1}\{\bar{T}(r, z ; s)\}$ where

$$
\bar{T}(r, z ; s)=\int_{0}^{\infty} \tilde{T}(k ; s) \tilde{I}(k) \mathrm{e}^{-\lambda_{p}(k ; s) z} \bar{f}(s) J_{0}(k r) k \mathrm{~d} k .
$$

Here, the functions $\tilde{T}(k ; s)$ and $\lambda_{p}(k ; s)$ are given by (11a) and (13b) with $\omega$ replaced by $i$.

In Figure 5 we compare the spatiotemporal evolution of the surface temperature profile $T(r, 0, t)$ by numerically inverting the Hankel and Laplace transforms in the case of Fourier and GK conduction. We have taken $f(t)=\exp (-t / \tau)$ where $\tau=10^{-7} \mathrm{~s}$ and $T_{0}=81 \mathrm{~K}$. Similar to when the heating function was harmonic in time, in this case we see that GK conduction also leads to a localisation of the temperature profile. Consequently, the maximum temperature induced by the pulse is an order of magnitude greater compared to the Fourier case. 


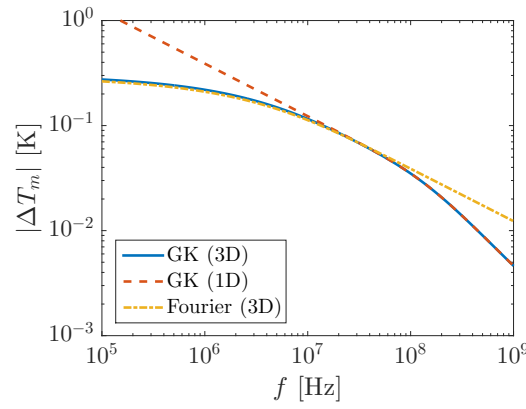

(a) $T_{0}=311 \mathrm{~K}$

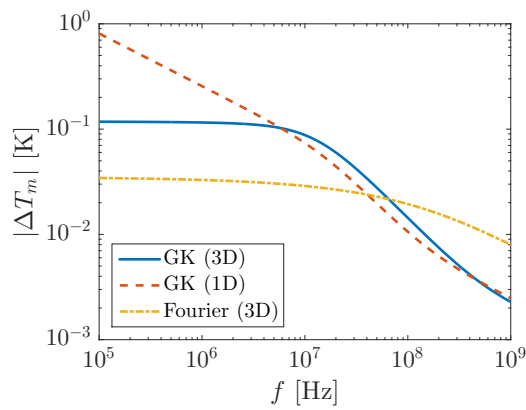

(c) $T_{0}=81 \mathrm{~K}$

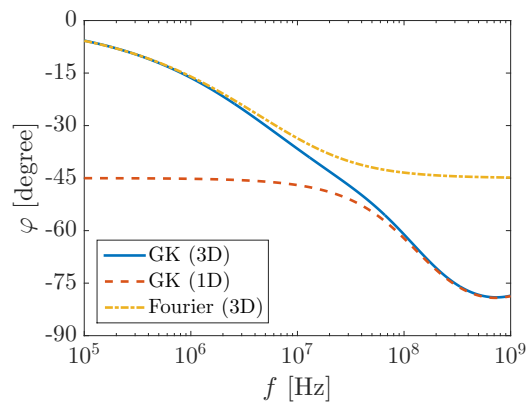

(b) $T_{0}=311 \mathrm{~K}$

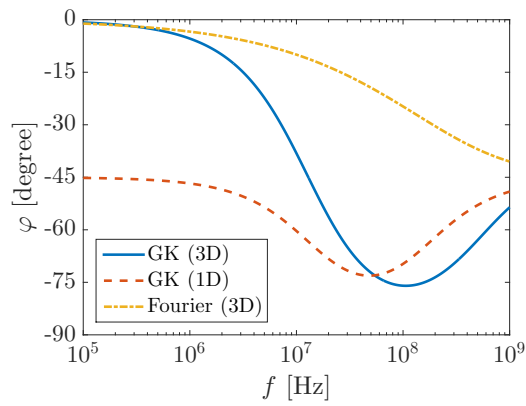

(d) $T_{0}=81 \mathrm{~K}$

Fig. 4 The frequency dependence of the amplitude $\left|\Delta T_{m}\right|$ and phase difference $\varphi$ of surface temperature oscillations measured with a probe beam computed using Fourier's law, the GK equation, and the one-dimensional GK equation.

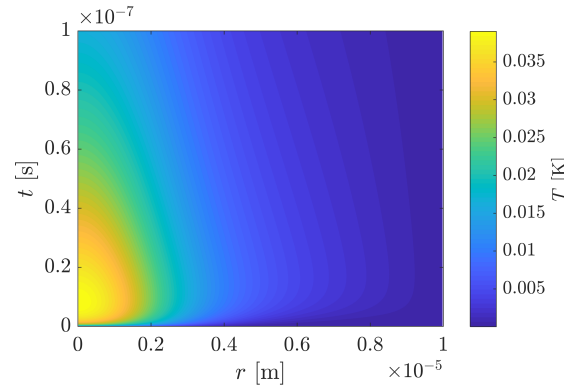

(a) Fourier conduction

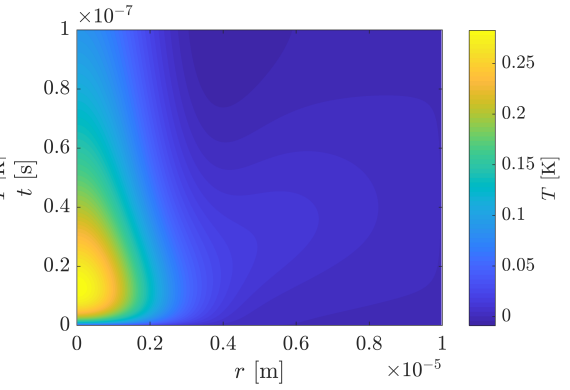

(b) GK conduction

Fig. 5 Surface temperature profiles induced by a laser pulse with an amplitude that varies in time according to $f(t)=\exp (-t / \tau)$ with $\tau=10^{-7} \mathrm{~s}$. The ambient temperature is $T_{0}=81 \mathrm{~K}$. 


\section{Conclusions}

We have used the GK equation to study heat conduction in thermoreflectance experiments. By exploiting the analogy between nanoheat heat transfer and viscoelastic wave propagation, analytical solutions to the problem were constructed. In the case of time-harmonic heating, we found there were three main regimes which can occur. For low frequencies, three-dimensional Fourier conduction was recovered. For intermediate frequencies, three-dimensional GK conduction occurs. Finally, for high frequencies, GK heat conduction becomes one-dimensional. The results also revealed that non-local effects due to normal phonon scattering introduce a thermal viscosity into the model. The additional resistance to heat flow leads to a localisation of the surface heating and higher temperatures compared to the Fourier case. However, the measurements of the surface temperature obtained using a probe beam, which are essentially the integrated product of the surface temperature and the beam profile, can result in values that are smaller than those predicted by Fourier's law. Thus, these measurements can miss the thermal localisation that occurs due to non-Fourier conduction.

We considered radially symmetric systems involving pump and probe beams with concentric circular cross sections. This allowed the solution to be written in terms of Hankel transforms. However, by using two-dimensional Fourier transforms, the solutions presented here could easily be adapted to handle a range of non-symmetric situations, such as those involving elliptical beams [26] or pump and probe beams with an offset [27]. These can provide deeper insights into the heat conduction mechanisms that occur at the nanoscale and further means of validating the GK equation or other non-Fourier conduction laws.

Acknowledgements This project has received funding from the European Union's Horizon 2020 research and innovation programme under grant agreement No 707658. MC acknowledges that the research leading to these results has received funding from 'la Caixa' Foundation. TM acknowledges financial support from the Ministerio de Ciencia e Innovación grant MTM2017-82317-P. The authors have been partially funded by the CERCA Programme of the Generalitat de Catalunya.

\section{References}

1. D. G. Cahill, W. K. Ford, K. E. Goodson, G. D. Mahan, A. Majumdar, H. J. Maris, R. Merlin, and S. R. Phillpot. Nanoscale thermal transport. J. Appl. Phys., 93(2):793-818, 2003.

2. D. G. Cahill, P. V. Braun, G. Chen, D. R. Clarke, S. Fan, K. E. Goodson, P. Keblinski, W. P. King, G. D. Mahan, A. Majumdar, H. J. Maris, S. R. Phillpot, E. Pop, and L. Shi. Nanoscale thermal transport. ii. 2003-2012. Appl. Phys. Rev., 1(1):011305, 2014.

3. M. Ashenghi, Y. K. Leung, S. S. Wong, and K. E. Goodson. Phonon-boundary scattering in thin silicon layers. Appl. Phys. Lett., 71(13):1798-1800, 1997.

4. D. Li, Y. Wu, P. Kim, L. Shi, P. Yang, and A. Majumdar. Thermal conductivity of individual silicon nanowires. Appl. Phys. Lett., 83(14):2934-2936, 2003.

5. W. Liu and M. Asheghi. Phonon-boundary scattering in ultrathin single-crystal silicon layers. Appl. Phys. Lett., 84(19):3819-3821, 2004. 
6. K. T. Regner, S. Majumdar, and J. A. Malen. Instrumentation of broadband frequency domain thermoreflectance for measuring thermal conductivity accumulation functions. Rev. Sci. Instrum., 84(6):064901, 2013.

7. K. T. Regner, D. P. Sellan, Z. Su, C. H. Amon, A. J. H. McGaughey, and J. A. Malen. Broadband phonon mean free path contributions to thermal conductivity measured using frequency domain thermoreflectance. Nat. Commun., 4:1640, 2013.

8. R. B. Wilson and D. G. Cahill. Anisotropic failure of Fourier theory in time-domain thermoreflectance experiments. Nat. Commun., 5:5075, 2014.

9. A. Beardo, M. G. Hennessy, L. Sendra, J. Camacho, T. G. Myers, J. Bafaluy, and F. X. Alvarez. Phonon hydrodynamics in frequency-domain thermoreflectance experiments. Phys. Rev. B, 101(7):075303, 2020.

10. C. Cattaneo. A form of heat conduction equation which eliminates the paradox of instantaneous propagation. Compte Rendus, 247(4):431-433, 1958.

11. R. A. Guyer and J. A. Krumhansl. Solution of the linearized phonon Boltzmann equation. Phys. Rev., 148(2):766, 1966.

12. R. A. Guyer and J. A. Krumhansl. Thermal conductivity, second sound, and phonon hydrodynamic phenomena in nonmetallic crystals. Phys. Rev., 148(2):778, 1966.

13. F. X. Alvarez, D. Jou, and A. Sellitto. Phonon hydrodynamics and phonon-boundary scattering in nanosystems. J. Appl. Phys., 105(1):014317, 2009.

14. D. Jou, J. Casas-Vázquez, and G. Lebon. Extended Irreversible Thermodynamics. Springer, fourth edition, 2010.

15. L. Zhang and X. Shang. Analytical solution to non-Fourier heat conduction as a laser beam irradiating on local surface of a semi-infinite medium. Int. J. Heat Mass Transfer, 85:772-780, 2015.

16. J. Maassen and M. Lundstrom. Modeling ballistic effects in frequency-dependent transient thermal transport using diffusion equations. J. Appl. Phys., 119(9):095102, 2016.

17. R. Kovács. Analytic solution of Guyer-Krumhansl equation for laser flash experiments. Int. J. Heat Mass Transfer, 127:631-636, 2018.

18. M. Calvo-Schwarzwälder, M. G. Hennessy, P. Torres, T. G. Myers, and F. X. Alvarez. A slip-based model for the size-dependent effective thermal conductivity of nanowires. Int. Comm. Heat Mass Transfer, 91:57-63, 2018.

19. M. Calvo-Schwarzwälder, M. G. Hennessy, P. Torres, T. G. Myers, and F. X. Alvarez. Effective thermal conductivity of rectangular nanowires based on phonon hydrodynamics. Int. J. Heat Mass Transfer, 126:1120-1128, 2018.

20. A. Sellitto, F. X. Alvarez, and D. Jou. Temperature dependence of boundary conditions in phonon hydrodynamics of smooth and rough nanowires. J. Appl. Phys., 107(11):114312, 2010.

21. C-Y Zhu, W You, and Z-Y Li. Nonlocal effects and slip heat flow in nanolayers. Scientific Reports, 7:9568, 2017.

22. U. Chouinard and N. Baddour. Matlab code for the discrete Hankel transform. J. Open Res. Software, 5(1), 2017.

23. D. G. Cahill. Analysis of heat flow in layered structures for time-domain thermoreflectance. Rev. Sci. Instrum., 75(12):5119-5122, 2004.

24. S. Both, B. Czél, T. Fülöp, G. Gróf, Á. Gyenis, R. Kovács, P. Ván, and J. Verhás. Deviation from the Fourier law in room-temperature heat pulse experiments. J. Non-Equilib. Thermodyn., 41(1):41-48, 2016.

25. P. Ván, A. Berezovski, T. Fülöp, Gy. Gróf, R. Kovács, Á. Lovas, and J. Verhás. GuyerKrumhansl-type heat conduction at room temperature. Europhys. Lett., 118(5):50005, 2017.

26. P. Jiang, X. Qian, and R. Yang. A new elliptical-beam method based on time-domain thermoreflectance (tdtr) to measure the in-plane anisotropic thermal conductivity and its comparison with the beam-offset method. Rev. Sci. Instrum., 89(9):094902, 2018.

27. J. P Feser and D. G. Cahill. Probing anisotropic heat transport using time-domain thermoreflectance with offset laser spots. Rev. Sci. Instrum., 83(10):104901, 2012. 